\title{
Why I despair at being a palliative medicine physician
}

Simon Wein, M.D. (D)

Pain and Palliative Care Service, Davidoff Cancer Center, Beilinson Hospital, Petach Tikva, Israel

Medicine like most professions is constantly changing. New treatments and new systems change our day-to-day practice. Purists might say that the relationship between the patient and the physician is unchanged - I disagree. From the bedside manner to telemedicine technique, I think the doctor-patient relationship has evolved for better and for worse.

Palliative Medicine in its original formulation helped people who were imminently dying. It was an outgrowth of the hospice movement in Europe. Later, as medicine became more complex, palliative medicine grew to include all patients who had an incurable disease and unrelieved symptoms. Thus, palliative medicine could be involved from the patient's first clinical presentation. Palliative Medicine is now recognized as a specialty in its own right in many countries around the world. The purview of Palliative Medicine covers all diseases, and not just cancer.

I wish to share my experience in Oncologic Palliative Medicine at a Comprehensive Cancer Center in Israel. In parallel with Oncologic Palliative Medicine, three other palliative areas have grown significantly - Psycho-oncology, Cancer Survivorship, and Integrative Medicine. These disciplines are formally recognized in major hospitals and comprehensive cancer centers around the world. These disciplines have journals and research literature that seek to provide an evidence base for their practice.

Alternative Medicine was a forerunner of Integrative Medicine. It does not have a scientific basis and its practitioners provide a limitless variety of unproven and disproven therapies to sick and suffering patients. They are motivated by many factors including spiritual belief systems, magic, financial reward, and the heroic (Wein, 2000). Every few years a new miraculous cure comes to market, with the same status and success rate as snake oil. Hope for health is a powerful motivator for patients to seek out "long-shot" treatments.

Cannabis is the latest claimant as an alternative cure of cancer and a universal palliator of symptoms. The claims are extraordinary and are well documented on the Internet. The actual evidence of clinical efficaciousness of cannabis is less than hope and hype would suggest (Boland et al., 2020). There are no high-quality randomized trials of cannabis for palliative care in cancer and there are unlikely to be. The main reason is the difficulty in designing a study to accurately measure the type and amount of cannabis (THC and CBD concentrations; smoked, vaporized, or oral) consumed and patient refusal to participate in randomized, placebo-controlled studies.

The growth and demand for cannabis have been dramatic and have transformed our palliative oncologic practice. Eight years ago there were no requests for cannabis in our out-patient clinic. Today, no less than $80 \%$ of our out-patient consultations involve a request for cannabis.

Several factors have driven this dramatic change, including government legislation, its psychotropic benefits, and belief in the healing power of cannabis.

The delivery of "medical" cannabis is complicated by this nexus of legislation and culture, and the tension between good and bad of the Janus-like cannabis plant. The battlefront is neither in the parliament nor in the media nor in laboratories. The battlefront is the physician's desk.

Government legislation in Israel has placed cannabis in a gray zone between a medication and a drug of abuse, with doctors as wardens. Cannabis has a limited medical benefit, but it is also a recreational drug with broad social acceptance. It is not paid for by the Government health budget, yet is available only via tightly controlled medical prescriptions, dispensed at regular pharmacies and paid for by the patients. In Israel, it has been decriminalized for personal use, though is not available commercially, like alcohol for example (Medical Cannabis Unit, 2021).

Cannabis does help cancer patients. In my experience, it mildly improves sleep, occasionally relieves nausea, somewhat improves appetite, alleviates mild pain, frequently relaxes, is mildly euphoric, and commonly neutralizes existential distress.

However, it is generally not effective in relieving moderate to severe cancer pain (Abrams, 2018); it is not an effective treatment for cancer cachexia; it is not effective for preventing vomiting from emetogenic chemotherapy; it does not treat major depression; and it does not alter the course of cancer. 
The last is an awful bugbear. Palliative medicine physicians practice according to evidence-based medicine or " $\mathrm{N}$ of 1 " trials. Many cancer patients come to us demanding extraordinary high doses of cannabis in order to distill Rick Simpson Oil which it is claimed that can cure cancer. There is no evidence that supports this claim. Yet desperate patients driven by hope want to try it as an alternative therapy.

We have queried the responsible body in the Ministry of Health. They state that this unproven use of cannabis is not one of their published indications and one just has to say, no, to the patients.

And therein lies the rub.

There is little value in explaining to desperately ill people that cannabis is an unproven therapy for cancer. Patients will routinely dissimulate in order to procure a minimum amount for the oil, which is much more than the maximum for symptom control. Anger, subterfuge, lies, wearying persistence, threats, complaints, doctor fatigue - often carry the day for the patient or their braying family.

By far the greatest need for cannabis is for its psychological benefit. Some patients use it every few hours to relax and "chill out" (Aviram et al., 2020). It is possibly similar to the way alcohol provides Dutch courage. When we suggest they are addicted, they vehemently deny it and say - "why shouldn't I use it, it does no harm, and only makes me feel good." When compared with other socially approved drugs such as alcohol and cigarettes, they might have a point.

Cannabis also helps to block distressing thoughts. Some patients specifically say that the thoughts are there but they are no longer disturbing. This appears to be a cognitive effect separate from euphoria.

The most egregious result of medical cannabis is the young people who have been cured of cancer who are rendered iatrogenic addicts. This is not a high point in a physician's career.

A significant amount of the medically prescribed cannabis is siphoned off for alternative uses: resale and recreational. Nobody not the police nor the relevant government bodies - has any idea of the amount of prescribed drug that is abused.

I am appalled to be placed in such an ethically compromising situation: to be "forced" to support unproven therapies; to be "forced" to supply a "medication" which will be used illegally; to be "forced" to supply a drug that will be on-sold for profit; and to inadvertently create iatrogenic addicts.

In the past couple of years, I have found the struggle to prescribe cannabis in a responsible, legal and ethical way enervating. No, this is an inadequate, pallid description. It is, in fact, the most horrible experience of my medical career. It has been humiliating. To see myself after 35 years of doctoring reduced to being a gatekeeper of this pseudo-medical drug (of addiction) and snake oil has burnt me. Colleagues laugh telling me "so what, just give them"; and "it's not your responsibility." I eventually found a balance with which I can live. It is not a perfect compromise, and every now and again I need a day off from work - a Mental Health Day.

A recent article makes the point that health organizations also bear a responsibility for the well-being of the professional. Therapy of the individual alone is insufficient to ameliorate suffering of the palliative care worker (Moreno-Milan et al., 2021).

I write this personal reflection, not in order to stop medical cannabis. Rather warn about the complexities of legislating medical cannabis. It might be that the particular mix of legislation and culture in Israel will prove to be less relevant in other societies.

It might be that it is more appropriate to make cannabis available like alcohol - individual responsibility with government restrictions.

\section{References}

Abrams DI (2018) The therapeutic effects of cannabis and cannabinoids: An update from the national academies of sciences, engineering and medicine report. Eur J Intern Med 49, 7-11.

Aviram J, Lewitus GM, Vysotski Y, et al. (2020) Short-term medical cannabis treatment regimens produced beneficial effects among palliative cancer patients. Pharmaceuticals (Basel) 13(12), 435.

Boland E, Bennett MI, Allgar V, et al. (2020) Cannabinoids for adult cancerrelated pain: Systemic review and meta-analysis. BMJ Supportive \& Palliative Care 10, 14-24.

Medical Cannabis Unit, Ministry of Health, Government of Israel. https:/ www.gov.il/he/departments/Units/cannabis_unit (accessed April 2021).

Moreno-Milan B, Breitbart B, Herreros B, et al. (2021) Psychological wellbeing if palliative care professionals: Who cares? Palliative \& Supportive Care. Published online 05 April, First View, 1-5.

Wein S (2000) Cancer, unproven therapies, and magic. Oncology (Huntingt) 9, 1345-1350. 